\title{
EXCITATION OF RESONANCES DUE TO THE SPACE CHARGE AND MAGNET ERRORS IN THE SNS RING *
}

\author{
A.V. Fedotov, N. Malitsky, Y. Papaphilippou, and J. Wei \\ BNL, Upton, NY 11973, USA \\ J. Holmes \\ ORNL, Oak Ridge, TN 37831-8218, USA
}

\begin{abstract}
The latest designs of high-intensity proton rings require minimal beam loss. The major cause of losses in a circular machine is the interplay of excited resonances, leading to particle diffusion. Such resonances could be driven by the space charge itself, magnet errors or a combined effect of the above. As a result, the goal of achieving low-level beam loss becomes especially challenging in the presence of both space charge and magnet imperfections. In this paper, we investigate the resonance excitation and their impact on the beam dynamics of the Spallation Neutron Source (SNS) ring, for various possible working points of the lattice.
\end{abstract}

\section{SPACE-CHARGE RESONANCES}

We refer to space-charge resonances as those which are driven by the space-charge potential rather than by the field potential of magnets. Their importance was first shown by Montague [1], for the dominant coupling resonance. This resonance can occur even for a linear lattice without any perturbations since it requires only a zero harmonic in the Fourier component of the density perturbation. Due to the fact that this resonance is a difference resonance such coupling can lead to a significant effect for a beam with unequal emittances. As a result, one has a typical recommendation of about one unit tune split for the high-intensity machines when the beam emittances in two transverse directions are significantly different. In the SNS case with correlated painting this can lead to an excessive halo even with equal emittances [2]. An important topic related to spacecharge coupling resonances is the possibility to drive instabilities of high-order collective beam modes [3]. The excitation of such modes and associated emittance growth were recently explored for typical beam parameters of a highintensity accumulator ring [4]. Moreover, other spacecharge induced resonances are possible when the driving force comes from the space-charge potential while the resonant harmonic $\mathrm{N}$ results from the magnet imperfections, or is related to the ring superperiodicity, which, in the latter case, leads to the space-charge induced superstructure resonances.

\section{EFFECT OF MAGNET ERRORS}

Machine resonances play a major role in halo formation [5]. Working points with tune split of half-integer or

\footnotetext{
* Work supported by the US Department of Energy
}

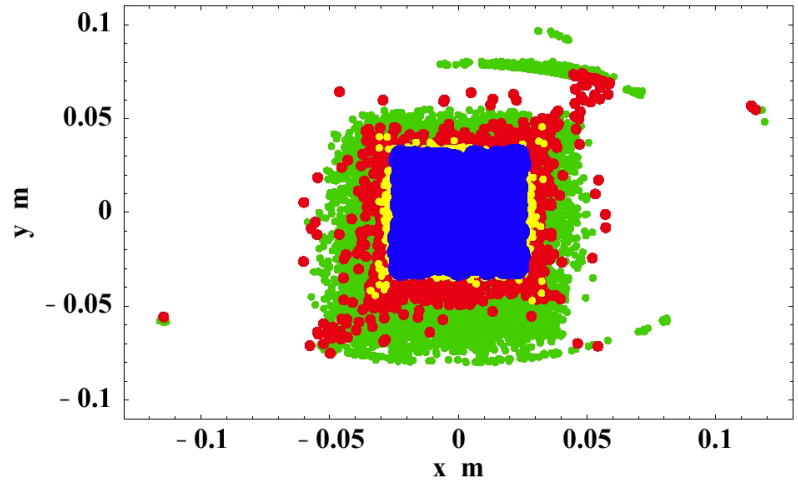

Figure 1: Blow-up of beam profile due to skew-quadrupole sum resonance in the presence of space charge: blue color (in the middle) - no space charge, no errors; yellow color space charge, no errors; red color - space charge, expected errors and quadrupole tilt $(0.2 \mathrm{mrad})$; green color - space charge, expected errors and quadrupole tilt (1 mrad).

more help to avoid strong coupling caused by the spacecharge forces and systematic magnet errors [6]. One of the SNS working points which satisfies such condition is $\left(Q_{x}, Q_{y}\right)=(6.3,5.8)$. As anticipated, the beam distribution stays stable under the influence of space-charge forces even when the systematic and random magnet fields errors of expected magnitude are included in simulations [2]. The situation dramatically changes when we introduce relatively small tilt $(0.2 \mathrm{mrad})$ in the quadrupoles. This effect is shown in Fig. 1 for the beam profile. In the absence of the space-charge, with the same tilt error in the quadrupoles, beam spreading disappears. After detailed study of such resonant behavior we confirmed that this is the manifestation of the sum resonance $Q_{x}+Q_{y}=12$. It takes place due to the combined effect of the space charge and skewquadrupole errors [2]. In the absence of the space charge the strength of introduced skew-quadrupole component (tilt of $0.2 \mathrm{mrad}$ ) was not sufficient for particles to be trapped into the resonance which requires $\Delta Q$ of 0.1 . However, the space charge depresses the tunes, and some particles are trapped into this sum resonance even for a relatively small skew-quadrupole components. Note that observed resonance is not the space-charge induced resonance since, in this case, it is driven by the skew-quadrupole field, and the role of the space charge is to push particles into the resonance. Such resonance can be corrected using the decoupling schemes. However, the vicinity of this dominant sum resonance makes the working point $\left(Q_{x}, Q_{y}\right)=(6.3,5.8)$ 
less attractive. Additional problems with this working point surfaced when we included the effect of the quadrupole fringe fields. As a result, we observed a significant beam loss due to the combined effect of the space charge and fringe fields.

One can see that finding the best choice of working point becomes very challenging for the SNS due to its special characteristics of a very large tune spread mainly associated with the space charge, chromaticity and magnet fringe fields.

\section{WORKING POINTS}

\subsection{Tune space}

The tunability of the new $248 \mathrm{~m}$ circumference lattice has been extensively explored [7]. It was shown that a tune-space is limited to one unit in the horizontal tune $Q_{x}=[6,7]$ and to approximately three units in the vertical tune $Q_{y}=[4,7]$ to avoid significant beatings of the beta functions. Such a limitation results due to the achromatic arc condition, which is required for dispersion-free straight sections, and optical matching.

The exploration of the resonance diagram for the SNS lattice [8]-[9] with superperiodicity four shows that the tune-space region less populated with the structure resonances is $Q_{x}=[5.5,6.5]$. Since lattice tunability studies showed good lattice matching only for $Q_{x}>6$, our horizontal tune space is essentially limited to $[6,6.5]$. If we want to avoid dangerous structure resonances up to the 4th order, our vertical tune space is also limited to $Q_{y}=[6,6.5]$. As a result, such a tune-space does not have any significant tune split flexibilities. Keeping in mind the advantages of the split-tune case, we retain the option of such a working point (up to one unit, for example, $\left.\left(Q_{x}, Q_{y}\right)=(6.23,5.24)\right)$. It will require correction of the structure resonances which we leave for future studies. Here, we limit our presentation to resonance studies of a tune region which is free at least from the structure resonances $\left(Q_{x}, Q_{y}\right)=[6,6.5]$.

We proceed by including non-structure resonances up to the 4 th order. The tune space for $\left(Q_{x}, Q_{y}\right)=[6,7]$ is shown in Fig. 2, with the black lines indicating imperfection non-structure resonances, the red lines indicating the 2nd order resonances, and the green lines showing the 3rd order structure resonances. Figure 2 also shows the expected tune spread of a $2 \mathrm{MW}$ beam, where the green tune footprint corresponds to the tune spread due to space charge alone, the red tune footprint includes an additional effect of momentum spread with $\Delta p / p=0.7 \%$, and the yellow tune footprint shows the combined effect of space charge and $\Delta p / p=1 \%$. In the SNS, $\Delta p / p=0.7 \%$ and $1 \%$ correspond to beam core and rf bucket acceptance, respectively. Note that in Fig. 2 the total tune spread due to space charge and chromaticity is not as big as one would expect using a simple linear superposition of these two effects.

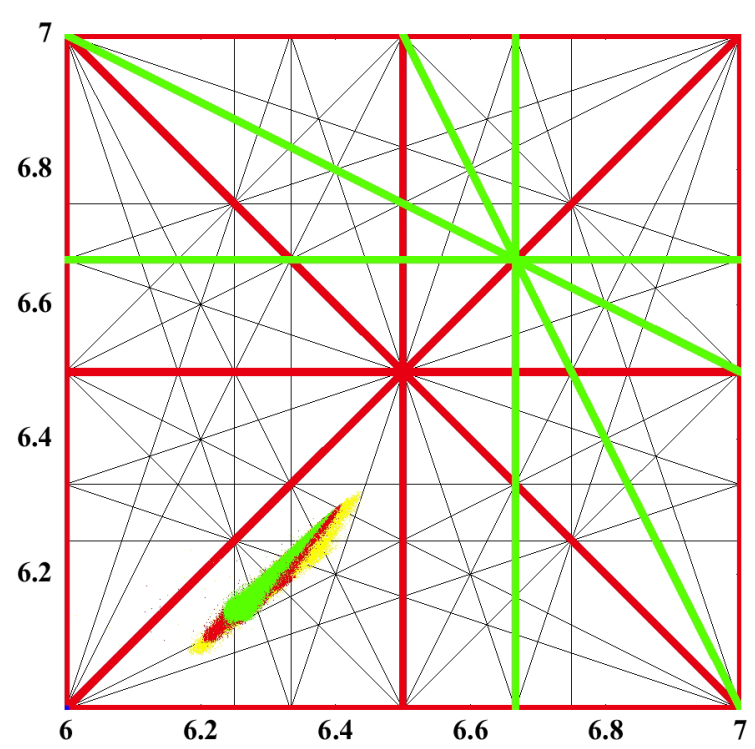

Figure 2: Working point $\left(Q_{x}, Q_{y}\right)=(6.4,6.3)$ with a tune footprint for a $2 \mathrm{MW}$ beam: green color represents tune spread due to space charge alone; red color also includes momentum spread of $0.7 \%$; yellow color shows combined effect of space charge and $\Delta p / p=1 \%$.

\subsection{Losses due to high order resonances}

Choosing the working point similar to the one shown in Fig. 2 gives us significant margin for intensity upgrade, and allows to paint to a smaller emittance (if only structure resonances need to be avoided). However, the beam will cross major 3rd order imperfection resonances such as normal sextupole $3 Q_{x}=19$ and skew-sextupole $2 Q_{x}+Q_{y}=19$. These resonances are not structural but can lead to significant losses if the excited terms are strong or the storage time is sufficiently long. In the SNS case, the injection time is only $1 \mathrm{msec}$, and the driving terms for these resonances are expected to be relatively small. We thus do not expect significant beam losses during $1 \mathrm{msec}$ injection period for this type of working point. These resonances are explored using the SNS simulation package of the UAL [2]. We introduce an additional source of sextupole errors by including chromatic sextupoles with the strength necessary to drive the chromaticity to zero. Beam halo at the end of 1052-turn injection is shown for various combinations of magnet errors in Fig. 3. To achieve losses at $10^{-4}$ level we require $10^{-3}$ losses at the primary scraper, which could be located in the acceptance region of $[240,280] \pi$ $\mathrm{mm}$ mrad. Figure 3 indicates that we have much larger halo in this region. However, the beam halo increase is not dramatic compared to the effect of space-charge only without magnet errors (shown in blue). Thus, we can paint to a smaller beam emittance to minimize beam halo to $10^{-3}$ level in the acceptance region of $[240,280] \pi \mathrm{mm}$ mrad. We can also adjust the working point to reduce an impact of skew-sextupole resonance, as well as try to correct it. 


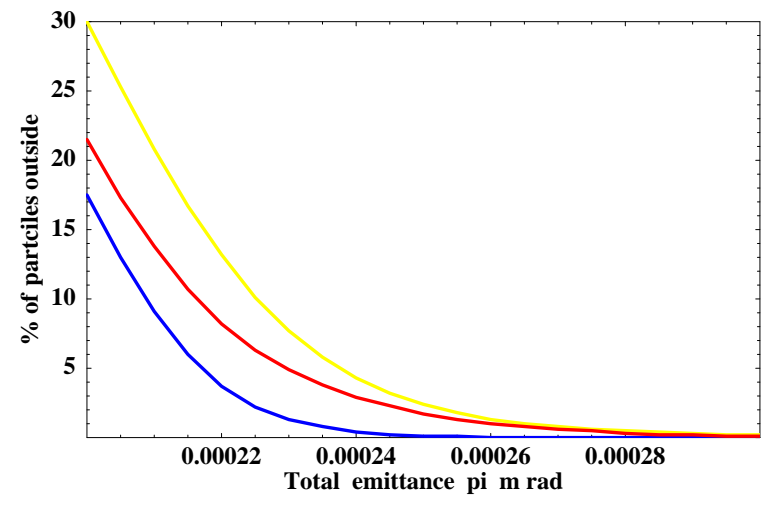

Figure 3: Beam halo due to expected magnet errors for $\left(Q_{x}, Q_{y}\right)=(6.4,6.3)$ : blue color shows halo due space charge alone; red color corresponds to the case of both systematic and random magnet field errors at a few units at $10^{-4}$ level, chromatic sextupoles and quadrupole fringe fields; yellow color shows an additional effect of $x, y$ misalingments of $0.5 \mathrm{~mm}$ and magnet tilt of $1 \mathrm{mrad}$.

\subsection{Low loss region}

Another choice of possible working point is the one which avoids the dangerous 3rd order resonances. Here, we discuss a working point which avoids both dangerous 3rd and 4 th order resonances. We can always increase the tunes of a working point slightly, if only the 3rd order resonances are a concern. As an example, we choose $\left(Q_{x}, Q_{y}\right)=$ $(6.23,6.2)$. Unfortunately, this point is quite close to the space-charge coupling resonance $2 Q_{x}-2 Q_{y}=0.06$, but otherwise our tune distribution will cross only the difference 3rd and 4th order resonances. The tune spread of a 2MW beam is shown in Fig. 4. We should also note that a working point similar to this is successfully used at PSR (although, with one unit split between the integer tunes), but at the highest intensities beam broadening is observed [10].

Usually, gradient errors are assumed to limit the intensity of the beam. This limit (incoherent space-charge tune shift) is based on the assumption of constant beam size. However, the beam size depends on the oscillation amplitudes of the individual particles, and if the gradient error causes these amplitudes to grow, the beam size also grows. Thus an approach of restricting the incoherent space-charge tune shift not to cross half-integer betatron line is not selfconsistent and is too conservative. The correct theory [11][12] indicates that one should use the "effective" tune shift rather than the incoherent tune shift when looking at the low-order betatron resonance conditions [5]. This theory seems to be in agreement with experimental studies at PSR [10]. It indicates that the space-charge limit is less restrictive, and that the choice of a working point close to the half-integer $(6.23,6.2)$ may be satisfactory even for highintensity SNS operation approaching $2 \mathrm{MW}$ beam. The main high-intensity limitation for this working point of the SNS is expected due to the half-integer systematic structure resonance and skew-quadrupole sum resonance. Some

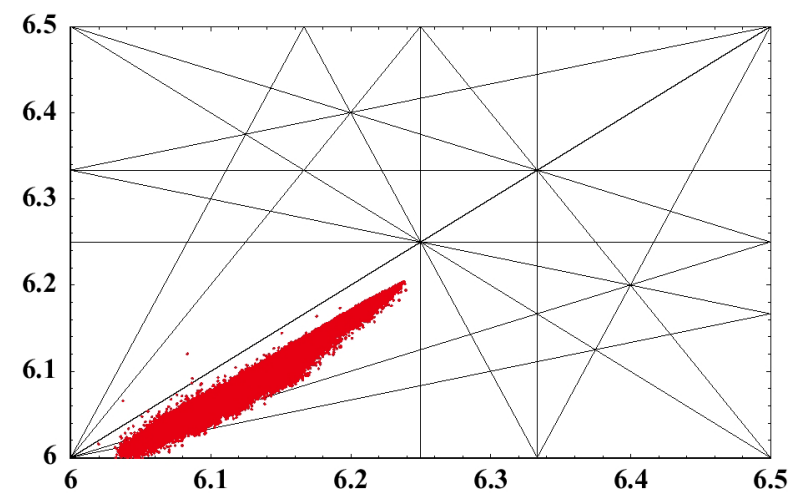

Figure 4: An example of $\left(Q_{x}, Q_{y}\right)=(6.23,6.2)$ working point. Tune spread (red) of a $2 \mathrm{MW}$ beam due to the combined effect of space-charge and $\Delta p / p=0.7 \%$.

effects which can modify the coherent resonance condition are currently under study. However, their impact is not expected to be dominant for the SNS beam parameters due to the very slow synchrotron motion. As a consequence of the coherent resonance, the beam response is quite different at resonance crossing compared to the one based on single particle dynamics. This topic requires dedicated discussion and will be presented elsewhere.

\section{ACKNOWLEDGMENT}

We thank G. Parzen, M. Blaskiewicz, Y.Y. Lee, S. Danilov and I. Hofmann for useful suggestions and discussions. We also thank the SNS team for discussions and support.

\section{REFERENCES}

[1] B.W. Montague, CERN Report 68-38 (1968).

[2] A.V. Fedotov, N. Malitsky and J. Wei, SNS/BNL Tech. Note 086 (2001).

[3] I. Hofmann, Phys. Rev. E 57, p. 4713 (1998).

[4] A.V. Fedotov, J. Holmes and R.L. Gluckstern, "Instabilities of high-order beam modes driven by space-charge coupling resonances", submitted to Phys. Rev. ST AB (2001).

[5] A.V. Fedotov et al., EPAC'00 (Vienna, Austria), p. 1289.

[6] J. Beebe-Wang et al., as in Ref [4], p. 1286.

[7] J. Holmes et al., "SNS Ring Optics Tuning", these proceedings.

[8] A.V. Fedotov, Y. Papaphilippou, J. Wei and J. Holmes, SNS/BNL Tech. Note 091 (2001).

[9] G. Parzen, SNS/BNL Tech. Note 090 (2001).

[10] J. Holmes et al., "Resonant Beam Response in the PSR Accumulator Ring", these proceedings; also Ref [4], p. 936.

[11] L. Smith, Int. Acc. Conf. (Dubna, Russia), p. 1232 (1963).

[12] F. Sacherer, Lawrence Rad. Lab Report UCRL 18454 (Ph.D. thesis, Univ. of California at Berkeley), 1968. 